\title{
An Unusual Case of Bilateral Meralgia Paresthetica Following Femoral Cannulations
}

\author{
Seong-il Oh, MD ${ }^{1,2}$, Eung Gyu Kim, MD', Sang Jin Kim, MD ${ }^{1,2}$
}

Meralgia paresthetica (MP) is a sensory mononeuropathy of the lateral femoral cutaneous nerve (LFCN). MP has rarely been reported after a femoral intervention approach. We report a case of bilateral meralgia paresthetica following bilateral femoral cannulation. A 64-year-old male received cardiac catheterization and treatment via a bilateral femoral vein. After cardiac catheterization, the patient presented with paresthesia in the anterolateral aspect of the bilateral thigh. After performing nerve conduction studies and electromyography, he was diagnosed as MP. Although a bilateral LFCN lesion following a femoral approach is very rare, MP might require caution regarding potential variations in LFCN when performing the femoral approach.

Key Words : Meralgia paresthetica; Femoral cannulation; Lateral femoral cutaneous nerve

Meralgia paresthetica (MP) is a sensory neuropathy of the lateral femoral cutaneous nerve (LFCN). It typically presents with painful paresthesia and numbness on the lateral thigh without weakness $[1,2]$. Though several contributing factors have been identified, the cause of its idiopathic form remains unclear. The most frequent causes of MP include obesity, pregnancy, pelvic mass lesions, external compression, and tight clothing, possibly leading to the entrapment of the LFCN under the inguinal ligament. It is

\footnotetext{
'Department of Neurology, Busan Paik Hospital, Inje University College of Medicine, Busan, Korea

${ }^{2}$ Dementia and Neurodegenerative Disease Research Center, Inje University, Gimhae, Korea

Received May 6, 2017; Revised July 25, 2017; Accepted July 26, 2017.

Correspondence to: Seong-il Oh, MD, PhD, Department of Neurology, Busan Paik Hospital, Inje University College of Medicine, 75 Bokji-ro, Busanjin-gu, Busan 47392, Korea.

Tel. 82.51.890.6130 Fax. 82.51.892.8811

E-mail: seongil.oh@gmail.com

This is an Open Access article distributed under the terms of the Creative Commons Attribution Non-Commercial License (http://creativecommons.org/licenses/by-nc/3.0) which permits unrestricted non-commercial use, distribution, and reproduction in any medium, provided the original work is properly cited.
}

commonly associated with diabetes [1]. In iatrogenic cases, laparoscopic surgery and orthopedic pelvic procedures have been associated with subsequent MP $[2,3]$. Anatomico-clinical studies have demonstrated high degrees of variability for the LFCN and have suggested an anatomical contribution to the pathogenesis of MP [4]. However, since the iatrogenic procedures known to have resulted in MP are usually unilateral, bilateral manifestations of MP are considered very rare [5].

Herein we report a patient with an unusual manifestation of bilateral meralgia paresthetica following a bilateral percutaneous femoral approach.

\section{CASE REPORT}

A 64-year-old man was referred for paresthesia on the lateral aspect of both thighs. He presented with a short history of several days of palpitation on arrival at our hospital. His initial electrocardiogram showed atrial fibrillation. Cardiac catheterization was performed to analyze three-dimensional electrophysiological studies. First punctures via the left femoral vein and the left subclavian vein were introduced to perform electro- 
physiological studies. His right femoral vein was cannulated with a 12 Fr sheath, and ablation was performed. Standard landmarks were visible, and the puncture was clean and uncomplicated. He underwent successful percutaneous treatment of the culprit lesion. After completion of the procedure, hemostasis was secured without complication. The day after the procedure, the sheath was removed; there was no significant hematoma present at the femoral puncture site. Symptoms started a few hours after percutaneous coronary intervention via the bilateral femoral artery. He complained of numbness and paresthesia over the anterolateral aspect of his bilateral thigh. Neurological examination confirmed an oval area of sensory deficit on both lateral thighs. A sensory nerve conduction study (NCS) of the LFCN was performed orthodromically with an active surface recording electrode $1 \mathrm{~cm}$ medial to the anterior-superior iliac spine. Sensory nerve action potentials (SNAPs) were decreased in amplitude bilaterally on the thigh. SNAPs on the right superficial peroneal nerve (SPN) decreased in amplitude but were preserved on the left SPN. Needle electromyography of the vastus lateralis, rectus femoris, and lumbar paraspinal muscles was unremarkable. He was otherwise well and was discharged to home the next day. At an out-patient clinic one week later, his complaints had decreased but follow-up NCS could not be performed.

\section{DISCUSSION}

Meralgia paresthetica is a syndrome of pain and/or dysesthesia in the anterolateral thigh, which is more common in obese subjects $[1,2]$. Meralgia paresthetica has been reported following local nerve damage during a subcutaneous injection, following spinal anesthesia, following pregnancy surgery, pelvic bone fracture, and after seatbelt trauma $[2,3,5]$. There is one unusual case report of it occurring following cardiac catheterization via the femoral artery and one case report of a bilateral MP occurring following cesarean section with epidural anesthesia [5]. However, despite these cases, a bilateral manifestation of MF following cardiac catheterizing is very rare.

Anatomico-clinical studies have demonstrated a variable course of the LFCN at its passage through the inguinal ligament [4]. The distance to the anterior superior iliac spine (ASIS) has been reported to range from a few millimeters to $7 \mathrm{~cm}$. Previous studies have suggested a relation between a small ASIS-LFCN distance and an elevated risk for the development of
MP, most likely due to increased mechanical stress to the LFCN caused not only by the bone but also by the tendon of the sartorius muscle and the inguinal ligament [4]. Thus, during local invasive procedures, it is important to realize that $25 \%$ of patients may present with anatomic variations of the LFTN.

When diagnosing MP, it is important to approach it clinically, with a detailed history and neurological examination [2]. Sensory nerve conduction and electromyography are important methods to assess and differentiate meralgia paresthetica. Recently, ultrasonography (US) as an adjunctive screening test in the diagnosis of compressive mononeuropathy has been shown to map morphologic changes of the nerve or a space-occupying mass at the lesion site. While US might be useful in evaluating morphologic changes or an aberrant course of the LFCN, it may be difficult to demonstrate the underlying cause in all patients with MP. Previous reports have suggested that US evaluation is useful for detecting a lesion or the abnormal pathway of the nerve around the ASIS $[2,4]$.

The majority of cases of MP have a favorable outcome, and $85 \%$ will resolve with conservative treatment [2]. In this case, we observed only a partial improvement for one week, but we could not observe the long-term clinical course. The prognosis of MP due to direct irreversible damage would be worse than that caused by transient damage of hematoma, local anesthesia and external compression $[1-3,5]$. To elucidate the prognosis of these patients, longitudinal clinical observations and additional cohort observations are needed.

Conservative treatments for MP include local analgesics, steroids, nonsteroidal anti-inflammatories, rest, wearing looser clothing, and reduction or elimination of aggravating factors. Although patients who do not respond to conservative treatment might be considered for an interventional management or surgical compression, there is insufficient evidence for a conclusive recommendation.

In this case, prevention can be tried by several methods based on the mechanism of development of MP due to LFCN damage after femoral cannulation. They may include the insertion of a vascular closure device after femoral cannulation [6], the avoidance of prolonged manual compression, the avoidance of unnecessary cannulation, and the avoidance of excessive local anesthesia. Nonetheless, conservative treatments for MP can be applied if symptoms occur even after applying these possible methods.

Our case report is subject to limitations. First, in this 
case, since the potential variations in LFCN were not clarified, it would be difficult to describe clinicoanatomical correlations in this patient. Also, the distance between the medial femoral cutaneous nerve and the femoral cannulation is usually shorter than the distance between LFCN and it [4, 7], medial femoral cutaneous nerve can be damaged more easily. Although NCS of medial femoral cutaneous nerve may be helpful in differentiating between LFCN and medial femoral cutaneous neuropathy, we could not performed medial femoral cutaneous nerve conduction study which is insufficient sensitivity and specificity. Even though, since this patient symptom is definitely confined to lateral femoral cutaneous nerve distribution, it is reasonable to do medial femoral cutaneous nerve conduction study to rule out completely. Second, somatosensory evoked potentials, which are elicited by stimulation of the LFCN below the ASIS, were not obtained. Therefore, MP occurring as a result of proximal changes in the LFCN cannot be excluded.

To the best of our knowledge, no case has been reported of bilateral meralgia paresthetica after bilateral femoral cannulation, and we recommend that potential anatomical variations in the LFCN should be noted and treated cautiously. Caution should further be applied because meralgia paresthetica could be a possible complication of femoral cannulation. Finally, diagnostic methods, including ultrasonography before an invasive femoral approach, and prevention methods might be helpful for differential diagnosis.

\section{References}

1. Parisi TJ, Mandrekar J, Dyck PJ, Klein CJ. Meralgia paresthetica: relation to obesity, advanced age, and diabetes mellitus, Neurology 2011;77:1538-1542

2. Patijn J, Mekhail N, Hayek S, Lataster A, van Kleef M, Van Zundert J. Meralgia Paresthetica, Pain practice: the official journal of World Institute of Pain 2011;11:302-308

3. Yamout B, Tayyim A, Farhat W. Meralgia paresthetica as a complication of laparoscopic cholecystectomy. Clin Neurol Neurosurg 1994;96:143-144

4. Moritz T, Prosch H, Berzaczy D, Happak W, Lieba-Samal D, Bernathova $\mathrm{M}$, et al. Common anatomical variation in patients with idiopathic meralgia paresthetica: a high resolution ultrasound case-control study. Pain Physician 2013;16:E287-293

5. Paul F, Zipp F. Bilateral meralgia paresthetica after cesarian section with epidural analgesia. J Peripher Nerv Syst 2006;11:9899

6. Duffin DC, Muhlestein JB, Allisson SB, Horne BD, Fowles RE, Sorensen $\mathrm{SG}$, et al. Femoral arterial puncture management after percutaneous coronary procedures: a comparison of clinical outcomes and patient satisfaction between manual compression and two different vascular closure devices. J Invasive Cardiol 2001;13:354-362

7. Oh SJ, Hatanaka Y, Ohira M, Kurokawa K, Claussen GC. Clinical utility of sensory nerve conduction of medial femoral cutaneous nerve. Muscle Nerve 2012;45:195-199 